\title{
Von verschiedenen Ligen, Regeln und Schiedsrichtern oder: Was das Theologiestudium mit Eishockey zu tun hat
}

Es mag für manche seltsam anmuten, das Theologiestudium in Beziehung zum Sport zu bringen. Diese Verwunderung dürfte noch verstärkt werden dadurch, dass hier ausgerechnet ein Bezug zum Eishockey hergestellt wird. Wenn schon eine Sportart, dann doch eher das anspruchsvolle Schach oder das noble Golf, nicht aber das vulgäre und aggressive Eishockey. Diese Bedenken - so wollen die folgenden spielerischen Ausführungen zeigen - sind aber völlig unbegründet. Die Parallelen zum Theologiestudium liegen nämlich nicht in erster Linie auf, sondern neben dem Spielfeld. Gewiss, auch von verwirrenden Kombinationen, harten Crosschecks, rasanten Flügelläufen, kläglichen Abschlussversuchen und effizientem Powerplay liesse sich manches erzählen. Doch noch interessanter scheint mir ein Blick auf das Regelwerk und das Schiedsrichterwesen zu sein.

\section{Zwei Ligen, zweierlei Schiedsrichter}

Im Sinne einer Vorbemerkung sieht sich der Verfasser gezwungen, näher darzulegen, warum er nicht das Fussball-, sondern das Eishockeyspiel einer näheren Betrachtung unterzieht. Dies geschieht einerseits deshalb, weil ansonsten leicht der Verdacht aufkommen könnte, hier werde im Stile eines billigen Plagiats pseudooriginelles Gedankengutin in leicht modifizierter Form wiederaufbereitet. Bekanntlich gehört es ja in manchen Kreisen beinahe schon zum guten Ton, mit Emphase die religiöses Dimension der schönsten Nebensache der Welt zu betonen und die Reihe der göttlichen Eigenschaften um das Attribut der «Rundheib» zu erweitern. Wichtiger noch ist aber der zweite Grund: Während die Regeln des Fussballspiels auch Nichteingeweihten innert vernünftiger Frist begreiflich zu machen sind, ist das beim Eishockey kaum zu schaffen. Entsprechend wichtig ist die Rolle des Schiedsrichtertrios, das eine klare Linie zu vertreten hat und gleichzeitig möglichst unauffällig bleiben soll. Beim Theologiestudium verhält sich all dies nicht anders, wobei sich hier das nicht zu unterschätzende Problem stellt, die zuständigen Schiedsrichter überhaupt identifizieren zu können. So einfach wie im Eishockey, wo sich jeder Zweifel angesichts der schwarzweiss gestreiften Dresses erübrigt, ist es beim Theologiestudium nicht. Die Schiedsrichter gehören nämlich zwei unterschiedlichen Organisationen an, die in einem nicht restlos geklärten Verhältnis zueinander stehen. Zusätzlich kompliziert wird die Lage durch die Existenz von zwei verschiedenen Ligen, in denen nach unterschiedlichen Regeln gespielt wird. Die Expertenmeinungen gehen auseinander, welches der beiden Reglemente das härtere sei. Brisant ist nun vor allem die Tatsache, dass die Schiedsrichter der einen 
Organisation wegen des akuten Schiedsrichtermangels Spiele beider Ligen leiten, während ihre Kollegen nur Spiele der eigenen Liga arbitrieren dürfen. Dass nicht zuletzt dieser Umstand für ein nicht ganz spannungsfreies Verhältnis der Referees unter einander sorgt, gehört aber ins Reich der puren Spekulation. Nichtsdestotrotz behaupten ewige Nörgler hartnäckig eine Konkurrenz der zwei Organisationen und weisen auf die unterschiedliche Qualifikation der Spielleiter hin. Bei den einen handle es sich um ausgebuffte Professionals, bei den anderen um zwar willige, aber oft überforderte Amateure. Auch das Argument, Letztere hätten samt und sonders eine (bisweilen glorreiche) Spielerkarriere hinter sich und seien deshalb sehr wohl ausreichend qualifiziert, wollen sie nicht gelten lassen, da ihrer Meinung nach die Anforderungen an Schiedsrichter nicht mit denen an Aktive vergleichbar seien.

\section{Von fälligen Reformen, erfolgten Reförmchen und ihren Gegnern}

Die skizzierte Situation schreit nach einer Klärung, doch ist eine Vereinigung der beiden Ligen und eine entsprechende Vereinheitlichung des Regelwerks im Moment nicht in Sicht. Wer davon überrascht ist, ist eingeladen, sein vielleicht etwas naives, weil zu vernünftiges Menschenbild einer Revision zu unterziehen. Wo es um die Wahrung von Besitzständen und die Erhaltung von ach so erhaltenswerten Traditionen geht, ist nichts nutzloser als vernünftige Argumente. Sei's wie es sei. Jedenfalls ist durchaus nachvollziehbar, dass Verteidiger des Status Quo - nicht selten selbst Laien-Referees - die bei einer Reform zu erwartende Professionalisierung des Schiedsrichterwesens fürchten. Sie betonen, dass sie ihren nebenamtlichen Job mit grosser Freude und Verantwortung und noch grösserem Engagement ausübten. Und wer schon Spiele unter ihrer Leitung absolviert hat, glaubt dies sofort. Aber die ihre Entmachtung fürchtenden Laienschiedsrichter sind nicht die einzigen, welche einer Reform skeptisch gegenüber stehen. Dem Vernehmen nach sollen nämlich auch die professionellen Schiedsrichter nicht daran interessiert sein, in Zukunft noch mehr Spiele leiten zu müssen, da sie an der Grenze ihrer Belastbarkeit angelangt seien. Sie hätten ja schliesslich noch andere Verpflichtungen, und das Leiten von Spielen sei nur ein Teil ihres Jobs, und nicht eben der reizvollste. Trotzdem geben die Befürworter einer Vereinheitlichung keine Ruhe. Sie führen unverdrossen das Argument ins Feld, die Zulassung von Laienschiedsrichtern sei im heutigen Spielbetrieb nicht mehr zeitgemäss und das Chaos im Schiedsrichterwesen gehe letztendlich auf Kosten der Spieler. Auch das zunehmende Desinteresse des Publikums spreche eine deutliche Sprache. Aber diese Stimmen verhallen meist ungehört. Zwar sind gerade vor kurzer Zeit einige Retuschen am Regelwerk der einen Liga vorgenommen worden, aber von einer Reform zu sprechen, wäre doch leicht übertrieben. Die Spielergewerkschaft jedenfalls hat auf die Regeländerungen wenig enthusiastisch reagiert: Immer noch mehr Spiele verhinderten die nötige Regeneration. Und die 
das Faktum nicht ausser acht zu lassen, dass die Spieler ihrerseits schon längst nicht mehr alle Vollprofis, sondern grossen Teils Amateure seien.

Die Kritik richtet sich vor allem auch gegen die jüngst beschlossenen Fähigkeitstests für die Aktiven. Wer gewisse Limiten nicht erfüllt, kann nämlich in Zukunft von Matches ausgeschlossen werden. Diese Massnahmen zur Nachwuchsförderung werfen einige Fragen auf. Hauptsächlich ist unklar, was das genaue Ziel der Massnahmen ist: Soll dadurch die notorische Abschlussschwäche der Stürmer behoben oder ganz einfach die mangelhafte Disziplin der Aktiven verbessert werden, wie einige argwöhnen? Für Gesprächsstoff ist jedenfalls gesorgt, und es bleibt abzuwarten, ob solche Massnahmen etwas zur Hebung des Spielniveaus beitragen. Die Spielfreude - so ist aus Spielerkreisen zu hören - werde dadurch bestimmt nicht gefördert. Ausgerechnet die Freude am Spiel ins Feld zu führen, mutet aber in Zeiten der global players reichlich naiv an, denn schliesslich ist der Eishockeysport schon längst den Kinderschuhen entwachsen und das spielerische Moment in den Hintergrund gerückt. Zuviel steht heute auf dem Spiel, als dass man sich den Forderungen nach Effizienz und Erfolg verschliessen könnte. Die Logik des Marktes macht auch vor den Sportstadien nicht halt. Diesen Binsenwahrheiten zum Trotz sind jedoch die nostalgischen Stimmten erstaunlicherweise noch nicht verstummt, die das mangelnde Spektakel und die Resultatfixiertheit des heutigen Spielbetriebs beklagen. Früher sei Eishockey zwar weniger athletisch, doch viel kreativer und auch schöner gewesen. Aus solchen Äusserungen ist unschwer das Pathos von Spielromantikern herauszuhören, die vom Selbstzweck des Spiels schwärmen und das Hohe Lied der Verspieltheit singen. Sie haben noch nicht realisiert, dass richtig Spass nur macht, was Erfolg bringt. Wer diese einfache Lektion gelernt hat - so die einhellige Meinung der Fachleute - ist auf dem besten Weg zu einer erfolgreichen Spielerlaufbahn. Der Ratschlag aus ihrem Mund an die Adresse der Aktiven kann daher nur lauten: Keine unnötigen Querpässe oder Dribblings, sondern konsequent den Abschluss suchen! Und vor allem: Volle Konzentration auf den eigenen Sport! Der Erfolg wird nicht auf sich warten lassen. Und ist der einmal da, fragt im Nachhinein bestimmt keiner mehr, wie er zustande gekommen ist.

- Christoph Amman studiert an der Theologischen Fakultät Zürich. 